\title{
Performance characteristics of the magnetic constrained layer damping
}

\author{
A. Baz and S. Poh \\ Mechanical Engineering Department, University of \\ Maryland, College Park, MD 20742, USA
}

Received 2 August 1999

Revised 21 February 2000

\begin{abstract}
A new class of surface damping treatment is proposed to provide effective means for attenuating undesirable structural vibrations. The proposed treatment relies in its operation on the use of smart damping treatments which consist of integrated arrays of constrained visco-elastic damping layers that are controlled passively by a specially arranged network of permanent magnets. The interaction between the magnets and the visco-elastic layers aims at enhancing the energy dissipation characteristics of the damping treatments. In this manner, it would be possible to manufacture structures that are light in weight which are also capable of meeting strict constraints on structural vibration when subjected to unavoidable disturbances.

Emphasis is placed here on introducing the concept and the basic performance characteristics of this new class of smart Magnetic Constrained Layer Damping (MCLD) treatments. Comparisons are also presented with conventional Passive Constrained Layer Damping (PCLD) in order to determine the merits and limitation of the MCLD treatments.
\end{abstract}

Keywords: Passive constrained layer damping, magnetic constrained layer damping and permanent magnets

\section{Introduction}

Passive Constrained Layer Damping (PCLD) treatments have been successfully utilized, for many years, to damp out the vibration of flexible structures ranging from simple beams to complex space structures [9]. However, the effectiveness of these treatments has been limited to a narrow operating range because of the significant variation of the damping material properties with temperature and frequency. Hence, treatments that are a hybrid between active and passive damping have been considered as viable alternatives to the conventional passive damping treatments. Such hybrid treatments aim at using one active control mechanism or another to augment the passive damping to compensate for its performance degradation with temperature or frequency. Among the commonly used hybrid treatments are the Passive Constrained Layer Damping with Shunted Networks (PCLD/SN) treatments [6], the Active Constrained Layer Damping (ACLD) treatments [3] and the Active Piezoelectric-Damping Composites (APDC) [5,8]. In the PCLD/SN treatments, a piezo-electric film is used to passively constrain the deformation of a visco-elastic layer that is bonded to a vibrating structure. The film is used also as a part of a shunting circuit which is actively tuned to improve the damping characteristics of the treatment over a wide operating range. Similar configuration is employed in the ACLD treatments. However, the piezo-film is actively strained in such a manner to enhance the shear deformation of the visco-elastic damping layer in response to the vibration of the base structure. In the APDC treatments, an array of piezo-ceramic rods embedded across the thickness of a visco-elastic polymeric matrix are electrically activated to control the compressional damping characteristics of the matrix which is directly bonded to the vibrating structure.

Therefore, in the three hybrid damping treatments described, one can identify three distinct damping augmentation mechanisms. In the PCLD/SN, the augmentation results from the energy dissipation in the shunted electric circuitry whereas in the ACLD and the APDC treatments, the augmentation is attributed to the enhanced shear and compressional deformations of the visco-elastic layers, respectively.

Although the PCLD/SN, ACLD and APDC treatments have proven to be very successful in damping out structural vibration, they require the use of piezoelectric films, amplifiers and control circuits. As simplicity, reliability, practicality and effectiveness are our ultimate goal in controlling the vibration and noise; the concept of the Magnetic Constrained Layer Damping (MCLD) is introduced. The MCLD eliminates the need 


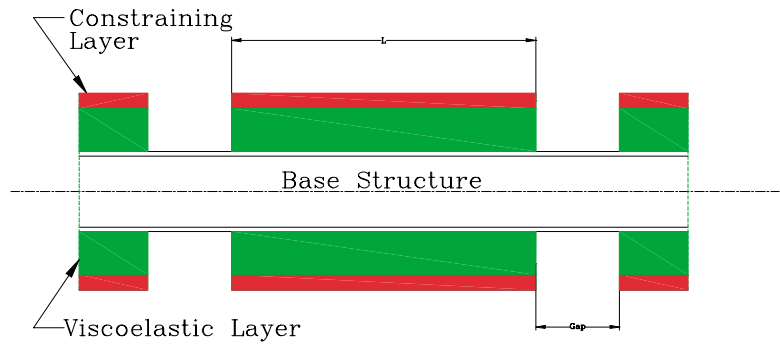

(a)

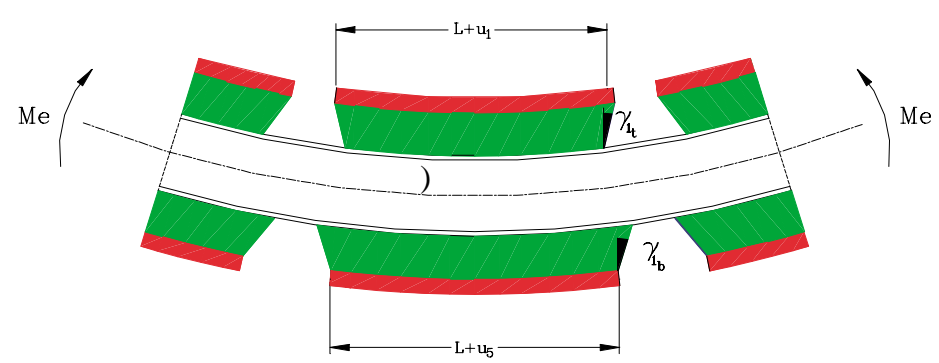

(b)

Fig. 1. Conventional multi-segment Passive Constrained Layer Damping (a) undeflected configuration and (b) deflected configuration.

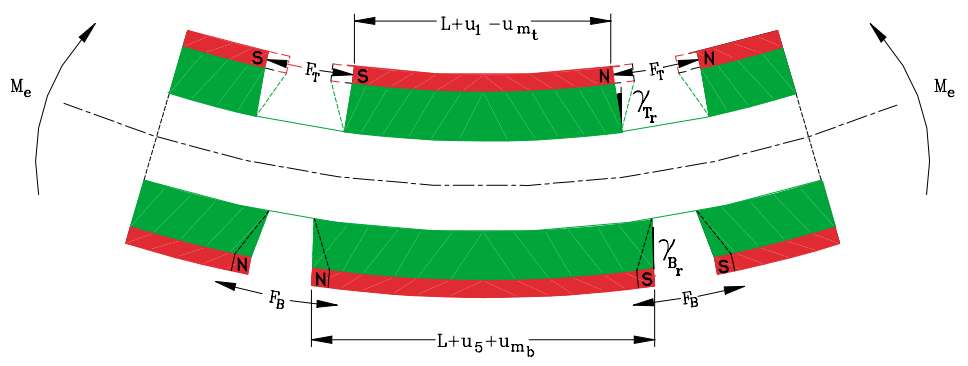

(a)

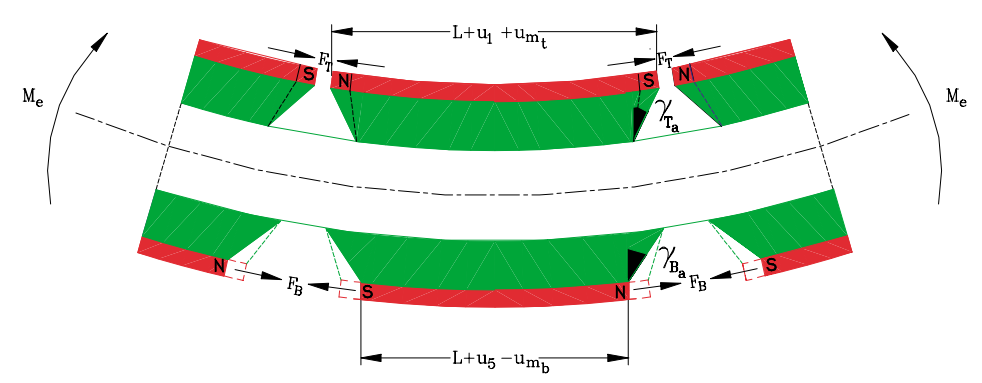

(b)

Fig. 2. Possible arrangements of the Magnetic Constrained Layer Damping (a) layers in repulsion and (b) layers in attraction. (PCLD - dashed lines and MCLD - solid lines).

for the piezo-films, associated circuitry as well as any external energy sources [1].

It is therefore our purpose to present here the concept of the Magnetic Constrained Layer Damping (MCLD) treatments and to evaluate its effectiveness in controlling the vibration of flexible beams as compared to conventional Passive Constrained Layer Damping (PCLD). Distributed-parameter modeling of the dynamics of 


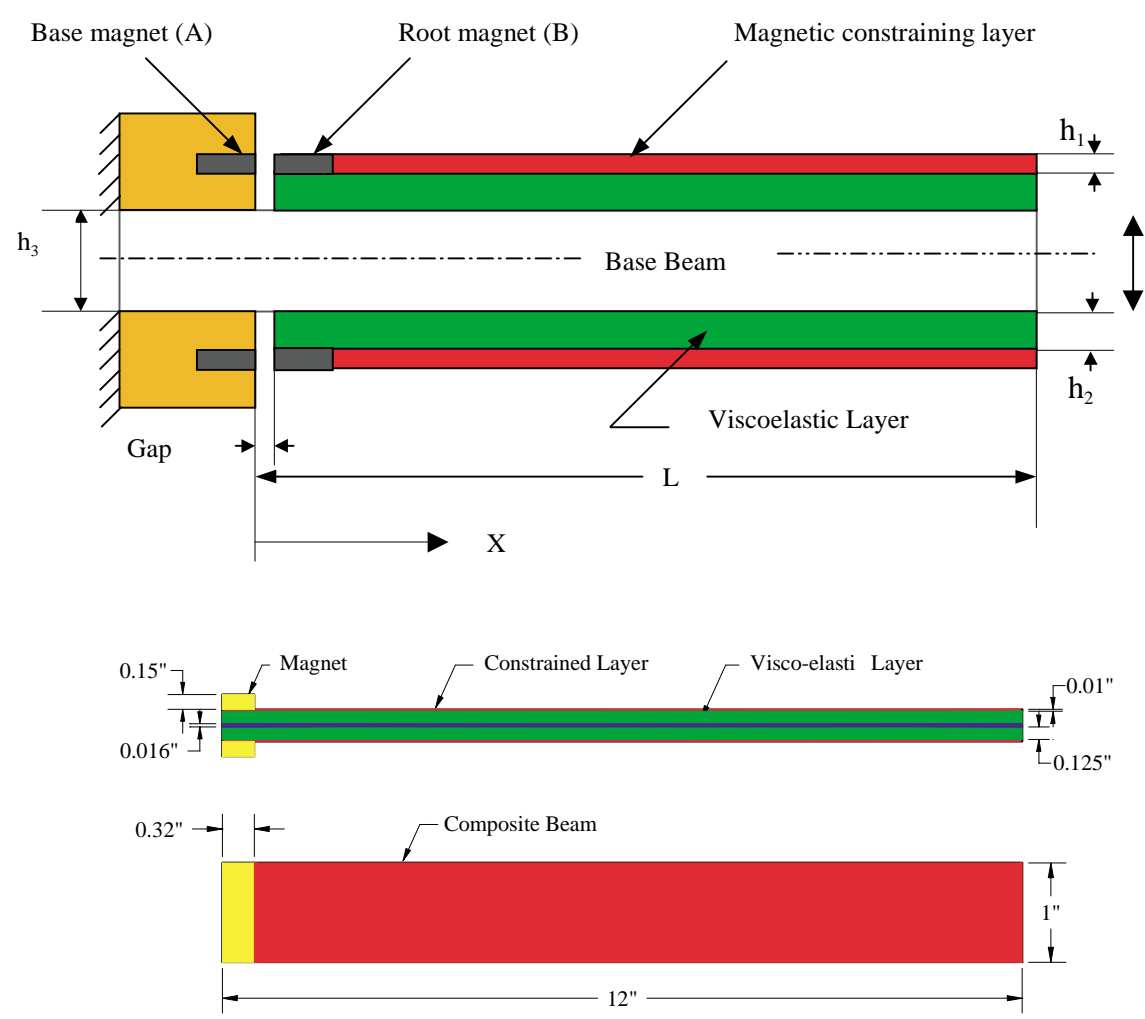

Fig. 3. Main geometric parameters of a full magnetic constrained layer damping treatment for cantilvered beam with base magnets.

beams treated with MCLD is presented by Baz [2] and finite element analysis of the magneto-elastic interactions in beams/MCLD system is developed by Ruzzene, Oh and Baz [7].

\section{Concept of magnetic constrained layer damping (MCLD)}

The concept of the MCLD can best be understood by considering first the multi-segment PCLD treatment shown in Fig. 1. The undeflected configuration of the structure/PCLD system is shown in Fig. 1(a) whereas Figure 1(b) shows the deflected configuration under the action of an external bending moment $M_{e}$. Due to such loading, shear strains of $\gamma_{T}$ and $\gamma_{B}$ are induced in the top and bottom viscoelastic layers respectively. Increasing these shear strains is essential to enhancing the energy dissipation characteristics of the damping treatment. A preferred way for increasing the shear strains is to replace the conventional constraining layers by magnetic constraining layers, which are properly arranged and designed.

Figure 2 shows two possible arrangements of the magnetic constraining layers where the inter-layer in- teraction is either in repulsion as in Fig. 2(a) or in attraction as in Fig. 2(b). Figure 2(a) shows that MCLD, with layers in repulsion, have strains $\gamma_{T r}$ and $\gamma_{B r}$ which are lower than the strains $\gamma_{T}$ and $\gamma_{B}$ of conventional PCLD treatments. Hence, it is not beneficial to arrange the magnetic layers in repulsion because of their low energy dissipation characteristics. This is in spite of the fact that such MCLD arrangement induces in-plane tensile loads in the base structure which tend to enhance its stiffness. However, it is evident that the resulting shear strains $\gamma_{T a}$ and $\gamma_{B a}$ of the attraction arrangement are much higher than the strains $\gamma_{T r}$ and $\gamma_{B r}$ of the repulsion arrangement. Note also that the strains $\gamma_{T a}$ and $\gamma_{B a}$ exceed the strains $\gamma_{T}$ and $\gamma_{B}$ of the PCLD treatment with conventional constraining layers. Therefore, significant improvement of the damping characteristics can be achieved by using MCLD treatments with magnetic layers in attraction. Note that the improved damping characteristics is attributed directly to the fact that the energy dissipation $E_{d}$ in the viscoelastic core is given by:

$$
E_{d}=\frac{1}{2} G^{\prime} \eta A \int_{0}^{L} \gamma^{2} \mathrm{~d} x
$$




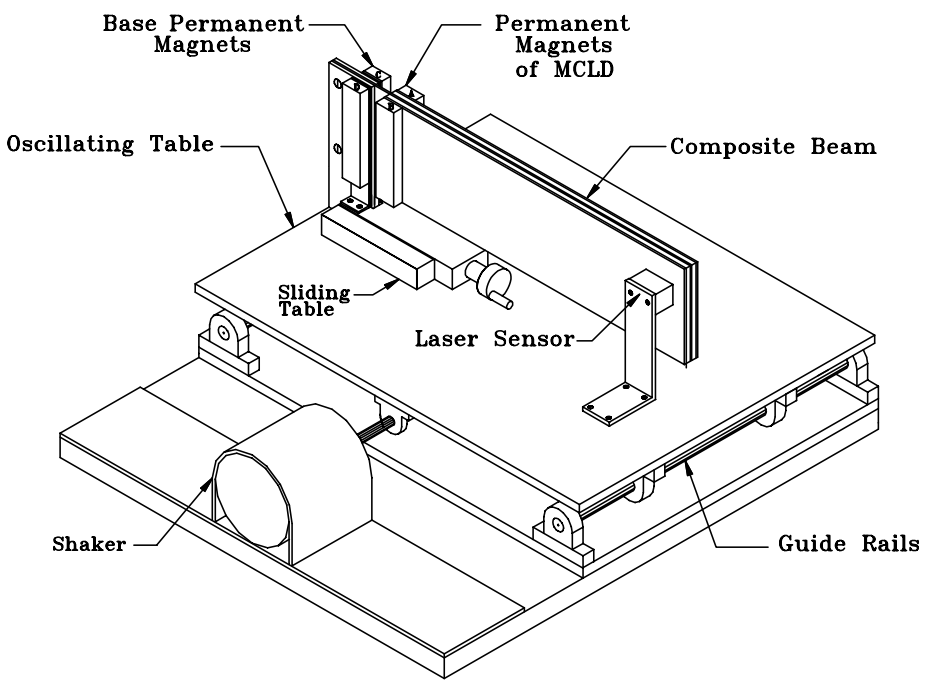

(a)

$$
\text { Laser sensor Beam }
$$

Shaker

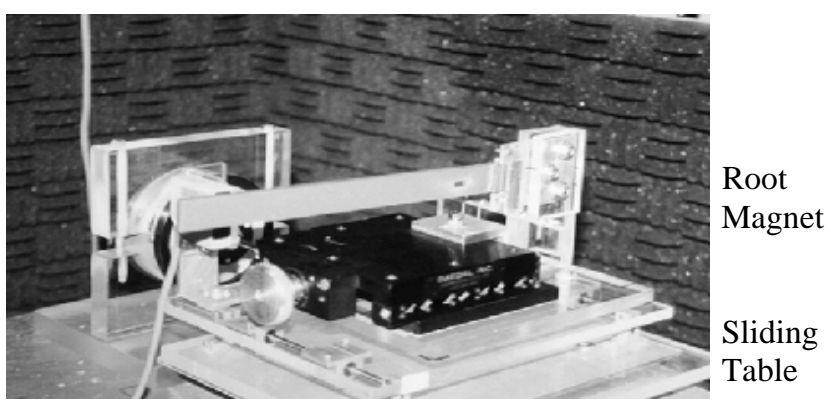

(b)

Fig. 4. The experimental set-up (a) schematic drawing, (b) photograph.

where $G^{\prime}=$ storage shear modulus, $\eta=$ loss factor, $A=$ surface area and $L=$ length of the viscoelastic core. Hence, increasing the shear strain $\gamma$ results in significant increase in the energy dissipated in the viscoelastic core. Such improved damping exists in the PCLD/SN, ACLD and APDC treatments but at the expense of the complexities associated with the use of piezo-sensors, piezo-actuators, control circuitry and/or external energy sources. Hence, the use of the MCLD improves the damping characteristics of conventional PCLD treatments and achieves such improved characteristics in a much simpler and efficient way than the PCLD/SN, ACLD and APDC treatments.

Hybrid configuration of the ACLD and MCLD can however be viable when the self-damped characteristics of the MCLD are to be enhanced by the use of the ACLD. With such a configuration, it would be pos- sible to compensate for performance degradation due to changes in the operating temperature and to improve/shape the frequency response characteristics of the composite assembly.

In what follows the improved damping characteristics of the MCLD are demonstrated in comparison with the performance of PCLD treatments.

\section{Performance characteristics of beam $/ \mathbf{m c l d}$ system}

\subsection{Materials}

The performance of the MCLD is determined experimentally using a cantilevered aluminum beam which is $30 \mathrm{~cm}$ long, $0.04 \mathrm{~cm}$ thick and $2.5 \mathrm{~cm}$ wide. The beam 

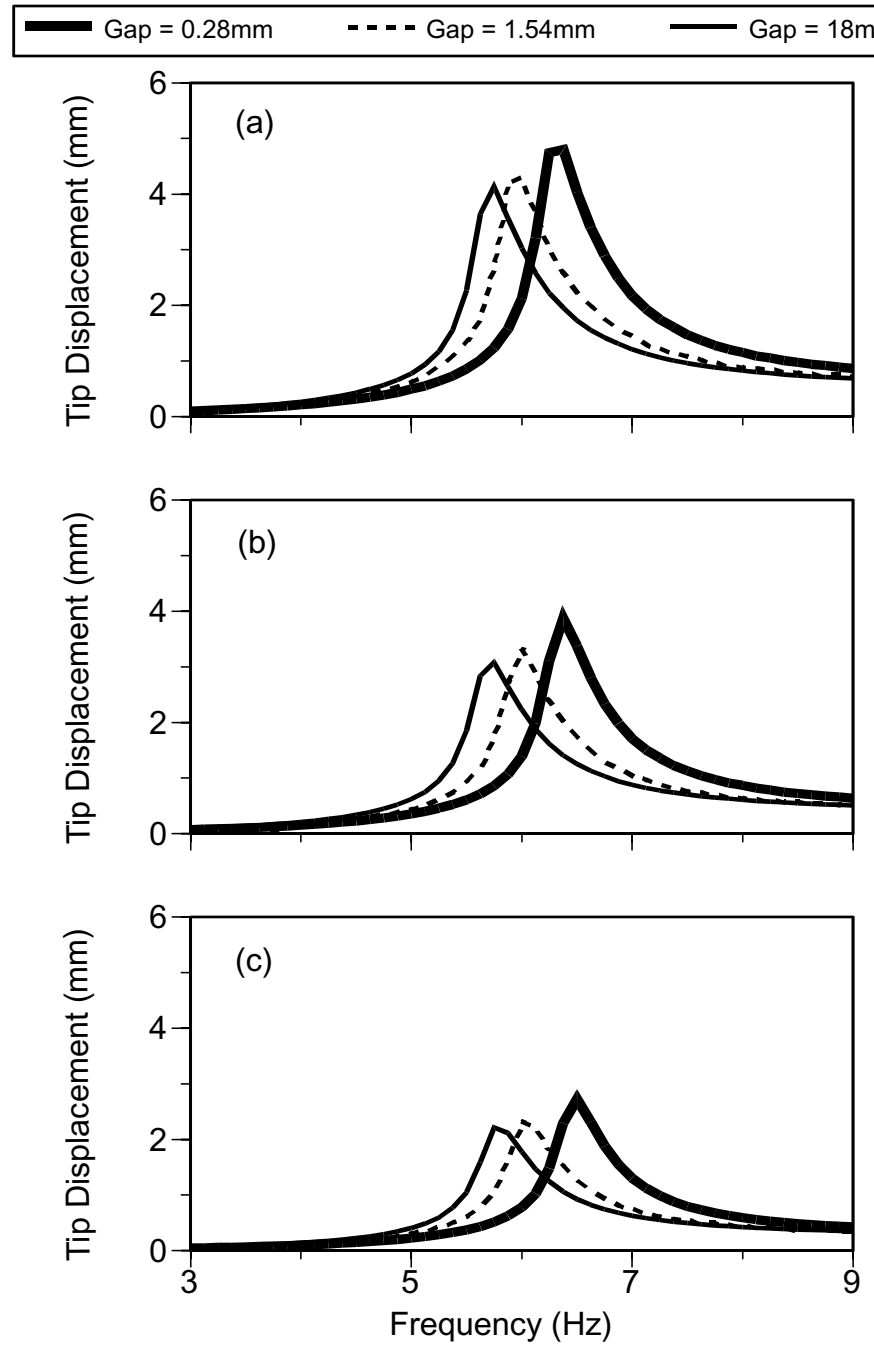

Fig. 5. Frequency response of beam tip at different gaps when magnets are arranged in repulsion for base displacement: (a) $0.23 \mathrm{~mm}$, (b) $0.17 \mathrm{~mm}$ and (c) $0.11 \mathrm{~mm}$

is fully treated with two viscoelastic damping layers that are $0.3125 \mathrm{~cm}$ thick. These layers have storage modulus $G^{\prime}=0.5 E 6 \mathrm{~N} / \mathrm{m}^{2}$, loss factor $\eta=0.4$ and density $=115 \mathrm{~kg} / \mathrm{m}^{3}$. The motion of the viscoelastic layers is constrained using aluminum layers which are $0.025 \mathrm{~cm}$ thick. The constraining layers are fitted with root magnets (B), as shown in Fig. 3. The magnets are made of neodymium blocks $(0.375 \times 0.8 \times 2.5 \mathrm{~cm})$ with residual induction $B_{r}=10,800$ Gauss and coercive force $H_{c}=9,300$ Oersteds. Similar permanent magnets (A) are placed in the base at an adjustable gap.

\subsection{Experimental set-up}

Figures 4(a) and 4(b) show a schematic drawing and a photograph of the experimental set-up used in testing the damping characteristics of the MCLD treatment. The beam/MCLD is clamped in a cantilevered configuration and is placed on an oscillating table which slides freely over guide rails. The table is connected to an electromagnetic shaker in order to subject the beam/MCLD system to a swept sinusoidal excitation. The beam response is monitored using a laser sensor placed at the beam tip. The response is recorded when the magnets are arranged in attraction or repulsion. In addition, the effects that the amplitude of base excitation and the gap between the interacting magnets have on the beam response are determined. A sliding table, mounted on the oscillating table, is used to adjust the gap between the magnets of the MCLD and the base magnets. 

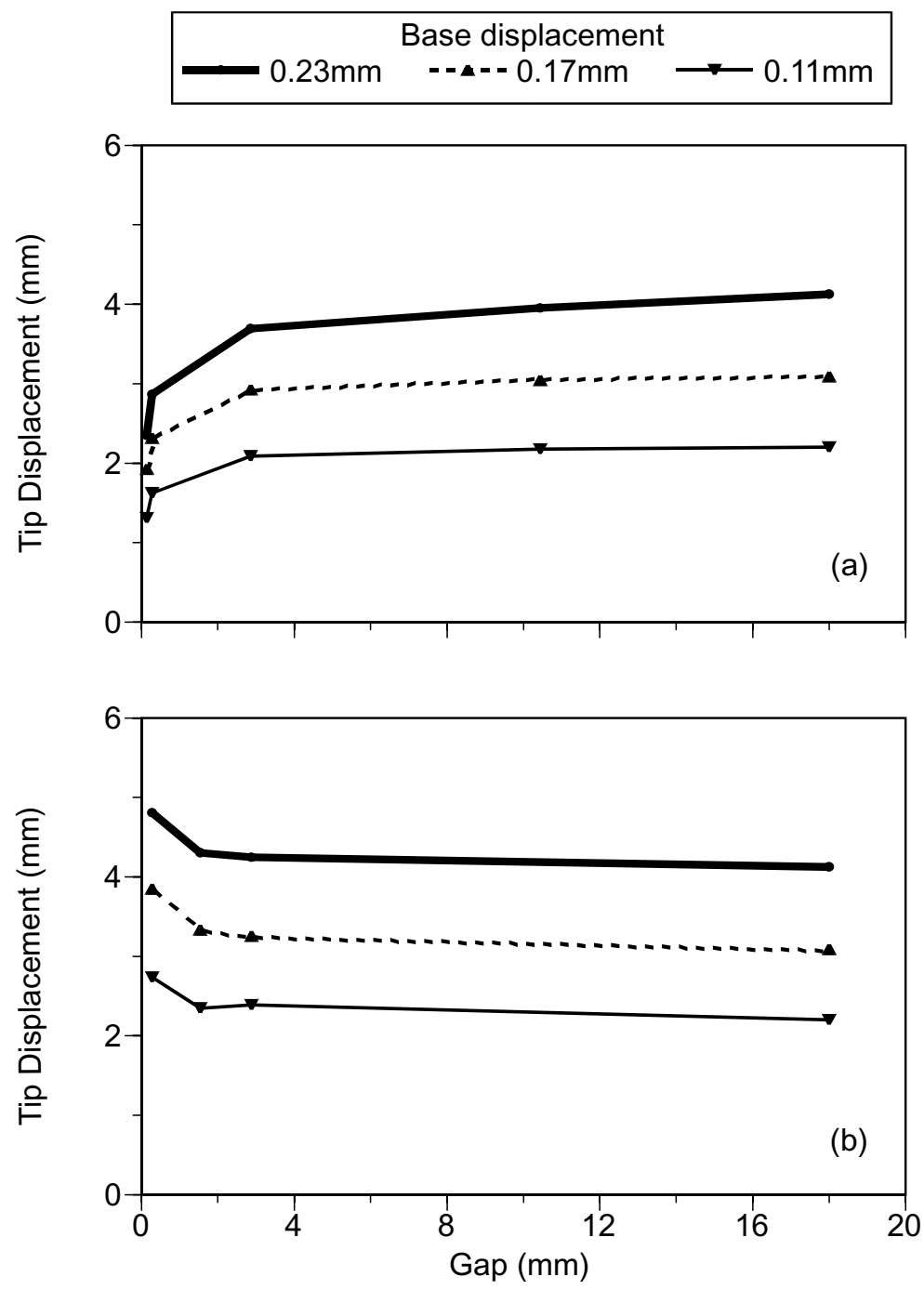

Fig. 6. Frequency response of beam tip at different gaps when magnets are arranged in attraction for base displacement: (b) $0.23 \mathrm{~mm}$, (b) $0.17 \mathrm{~mm}$ and (c) $0.11 \mathrm{~mm}$

\subsection{Experimental results}

\subsubsection{Full treatment with magnets in base and in constraining layers}

Figure 5 shows the frequency response of the beam/MCLD for different gaps and amplitudes of base excitation when the magnets are arranged in repulsion. Note that the beam vibration, as measured by its tip displacement, increases as the gap is decreased. Such adverse effects are attributed primarily to the reduction of the damping characteristics of the MCLD as described in Fig. 2(a). These effects occurred in spite of the stiffening of the beam/MCLD system that manifested itself clearly by the considerable increase of the first natural frequency of the system. The resulting frequency increase is attributed to the induced in-plane magnetic loads. Figure 5 indicates also that increasing the amplitude of base excitation increases the amplitude of vibration of the beam.

It is important here to note that when the gap between the magnets reaches $18 \mathrm{~mm}$, the magnetic forces become very small and the MCLD treatment converges to the conventional PCLD treatment. Therefore, the results obtained indicate that the PCLD outperforms the MCLD with magnets arranged in repulsion. Table 1 summarizes the effect of the gap and amplitude of base displacement on the damping ratio of the MCLD at the first natural frequency as obtained by using the half-power method. 

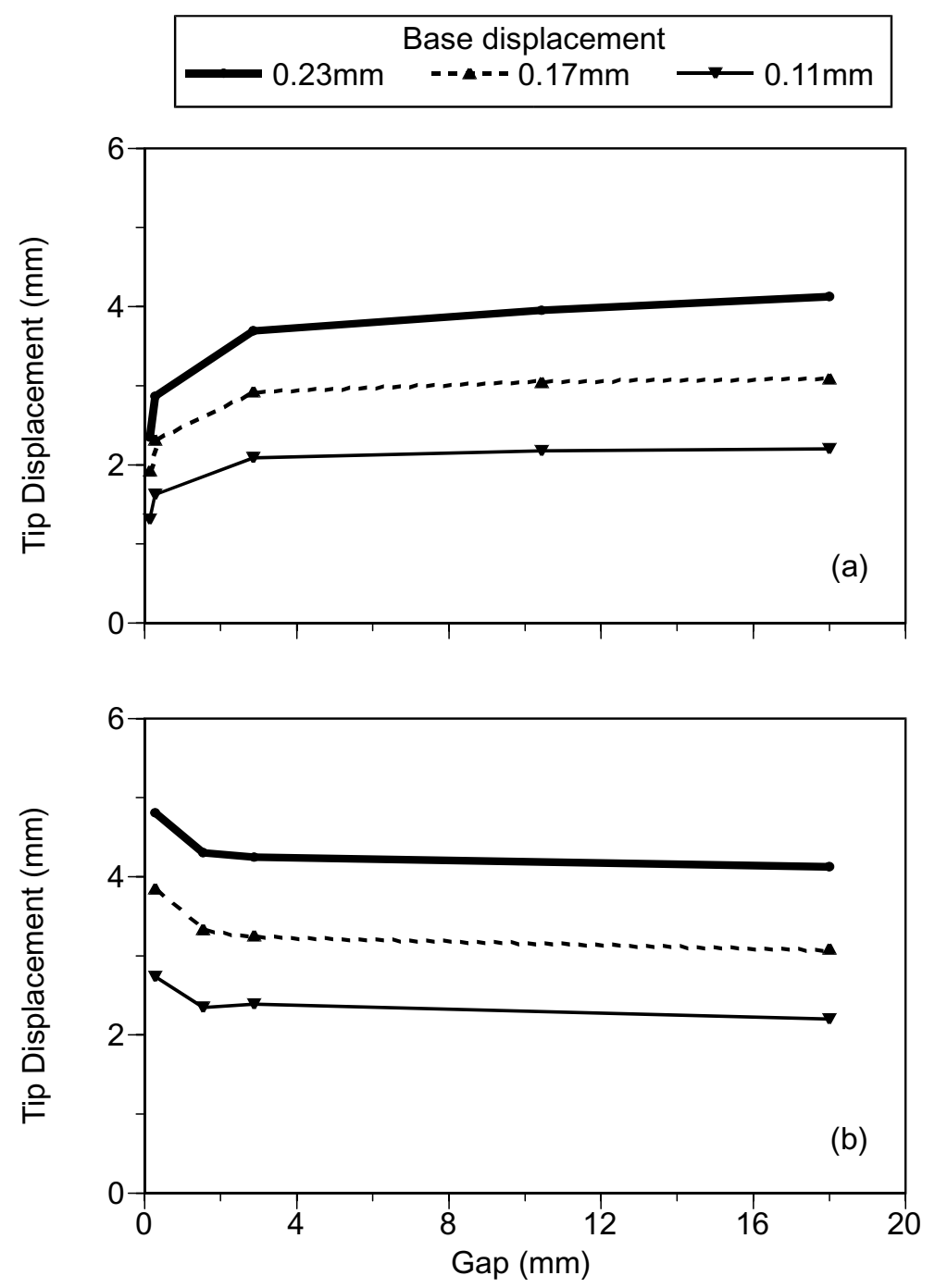

Fig. 7. Effect of magnet arrangement on performance of MCLD: (a) Magnets in attraction and (b) Magnets in repulsion.

Table 1

Effect of gap and base displacement on damping ratio (magnets in repulsion)

\begin{tabular}{llcl}
\hline Gap $(\mathrm{mm})$ & \multicolumn{3}{c}{ Base displac. $(\mathrm{mm})$} \\
\hline & 0.23 & 0.17 & 0.11 \\
18 & 0.047 & 0.0378 & 0.0407 \\
1.54 & 0.044 & 0.0388 & 0.0388 \\
0.28 & 0.037 & 0.0317 & 0.034 \\
\hline
\end{tabular}

The results displayed in Table 1 indicate that reducing the gap (i.e. increasing the magnetic interactions) results in lowering the modal damping ratios. This emphasizes clearly the ineffectiveness of arranging the magnets in repulsion. Note, however, that increasing the amplitude of base displacement results in improving the damping ratio of the MCLD. However, the con- verse becomes true when the magnets of the MCLD are arranged in attraction as shown in Fig. 6 for different gaps and amplitudes of base excitation. Significant attenuation of the beam vibration is observed when the gap between the magnets is decreased and the when the amplitude of the base excitation is increased. Such improved performance is attributed to the enhanced damping characteristics described in Fig. 2(b). Furthermore, the observed attenuation is achieved in spite of the softening of the beam/MCLD system due to the in-plane compressive magnetic loads.

Table 2 summarizes the effect of the gap and amplitude of base displacement on the damping ratio of the MCLD at the first natural frequency.

From Tables 1 and 2, it is evident that arranging the magnets in attraction results in significant increase in 


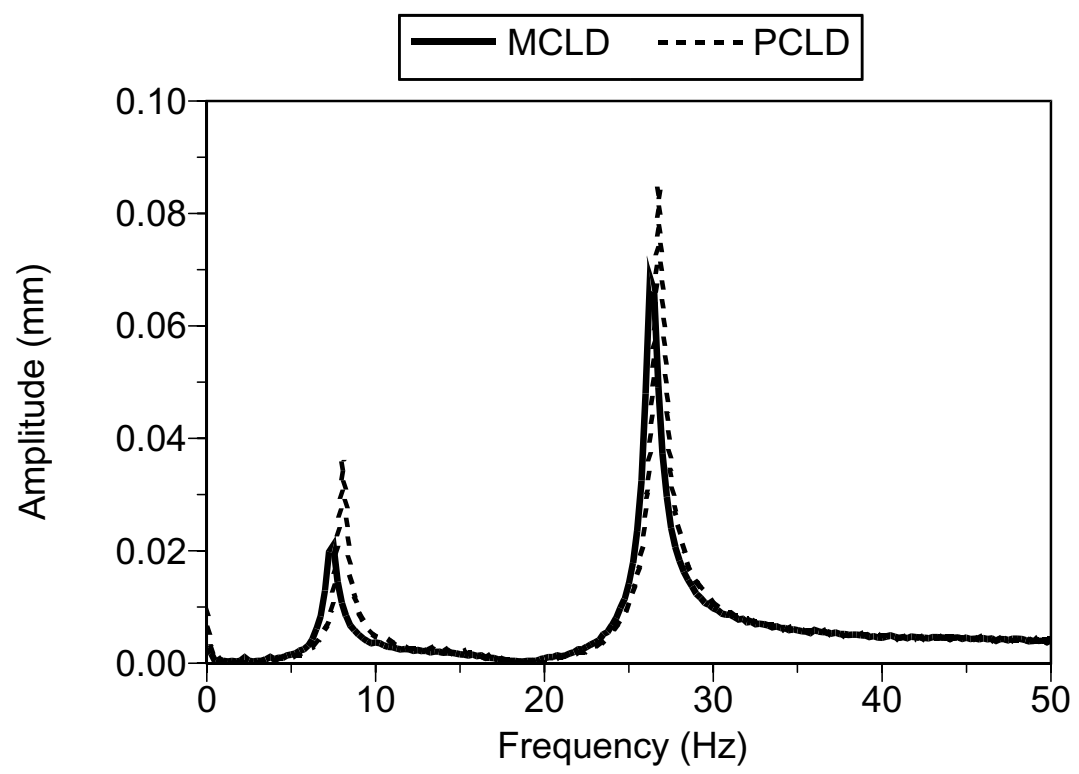

Fig. 8. Effect of partial treatment on performance of MCLD.

Table 2

Effect of gap and base displacement on damping ratio (magnets in attraction)

\begin{tabular}{llcl}
\hline Gap $(\mathrm{mm})$ & \multicolumn{3}{c}{ Base displac. $(\mathrm{mm})$} \\
\hline & 0.23 & 0.17 & 0.11 \\
18 & 0.035 & 0.040 & 0.035 \\
0.28 & 0.053 & 0.053 & 0.050 \\
0.14 & 0.066 & 0.066 & 0.066 \\
\hline
\end{tabular}

the modal damping ratios emphasizing the importance of the damping mechanism in attenuating the structural vibration. Note that such improvement has occurred in spite of the softening effect of the structure which is demonstrated clearly by the shift of the natural frequency to the left as the gap is decreased (Fig. 6).

Figure 7 summarizes the effect of the gap, the base excitation and the arrangement of the magnets on the performance of the MCLD treatment. The figure indicates that MCLD treatments with magnets in attraction is much superior than the conventional PCLD treatments particularly when the gap is reduced and the excitation level is increased.

\subsubsection{Partial treatment with magnets in base and in constraining layers}

Figures 5 and 6 show that the damping effect produced by the MCLD treatment is limited to the first mode of vibration of the beam. However, improved performance of the MCLD treatment is achieved, over a broader frequency range, when the treatment of the beam become partial as indicated in Fig. 8. In this case
Table 3

Effect of gap and base displacement on damping ratio (magnets in base only)

\begin{tabular}{llcl}
\hline Gap $(\mathrm{mm})$ & \multicolumn{3}{c}{ Base displac. $(\mathrm{mm})$} \\
\hline & 0.23 & 0.17 & 0.11 \\
18 & 0.040 & 0.040 & 0.040 \\
0.28 & 0.060 & 0.060 & 0.060 \\
0.14 & 0.086 & 0.086 & 0.086 \\
\hline
\end{tabular}

of partial treatment, the MCLD treatment extends over half the beam span starting from its fixed end.

\subsubsection{Full treatment with magnets in base only}

So far, all our presentation has concentrated on the use of interacting magnetic constraining layers. However, to maintain the simplicity of the MCLD treatment; we only need magnets in the base to interact with ferromagnetic constraining layers. The interaction forces in that case are always attraction forces which are found essential for enhancing the damping characteristics of the treatment. In this manner, the weight and cost of the treatment are reduced considerably.

Figure 9 shows the frequency response of the beam for different gaps and amplitudes of base excitation. It is evident that the performance is comparable with that of MCLD treatments with magnets in base and on the constraining layers which is shown in Fig. 6. Figure 10 summarizes the effect of the gap and the base excitation on the performance of the MCLD treatment.

Also, Table 3 summarizes the effect of the gap and amplitude of base displacement on the damping ratio of the MCLD at the first natural frequency. 


Gap $=0.10 \mathrm{~mm} \quad----$ Gap $=0.44 \mathrm{~mm} \quad \longrightarrow$ Gap $=5.22 \mathrm{~mm}$
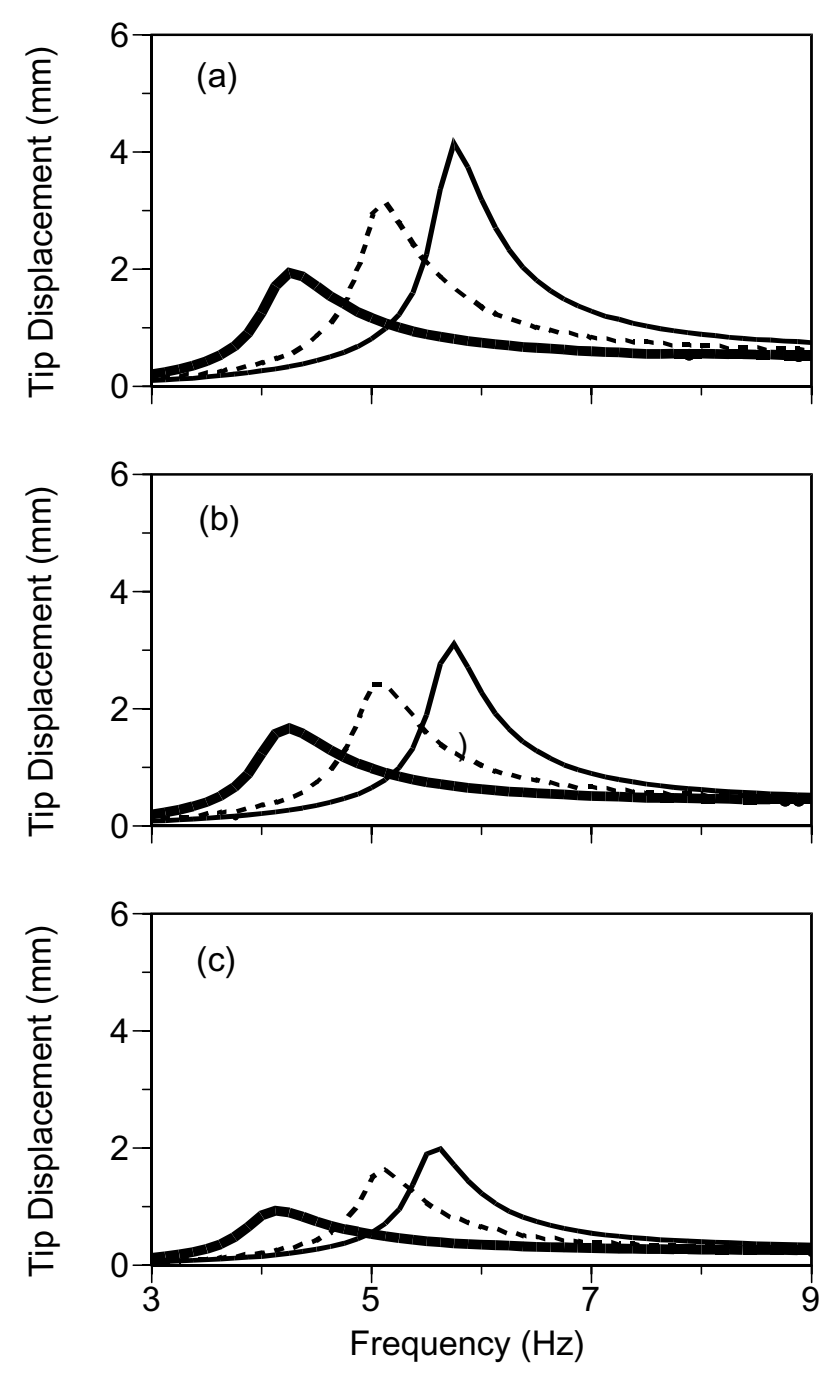

Fig. 9. Frequency response of beam tip at different gaps with base magnets and ferromagnetic constraining layers for base displacement: (c) $0.23 \mathrm{~mm}$, (b) $0.17 \mathrm{~mm}$ and (c) $0.11 \mathrm{~mm}$.

The displayed results emphasize the potential of the MCLD in enhancing the damping characteristics of beams over broad frequency range without the need for electronic sensors, actuators or control circuitry.

\section{Conclusions}

This paper has presented a new class of Magnetic Constrained Layer Damping (MCLD) treatments which relies in its operation on array of viscoelastic damping layers that are controlled completely passively by a specially arranged network of permanent magnets. The proposed MCLD treatments enhances the damping characteristics of conventional Passive Constrained Layer Damping (PCLD) treatments without the need for any electronic sensors, control circuitry or external energy sources. This is in contrast to the Active Constrained Layer Damping (ACLD) treatments and the Passive Constrained Layer Damping with Shunted Networks (PCLD/SN) which require extensive electronics for their effective operation. Such excellent feature of the MCLD makes its operation simple, reliable and efficient as compared to other surface damping treatments. 


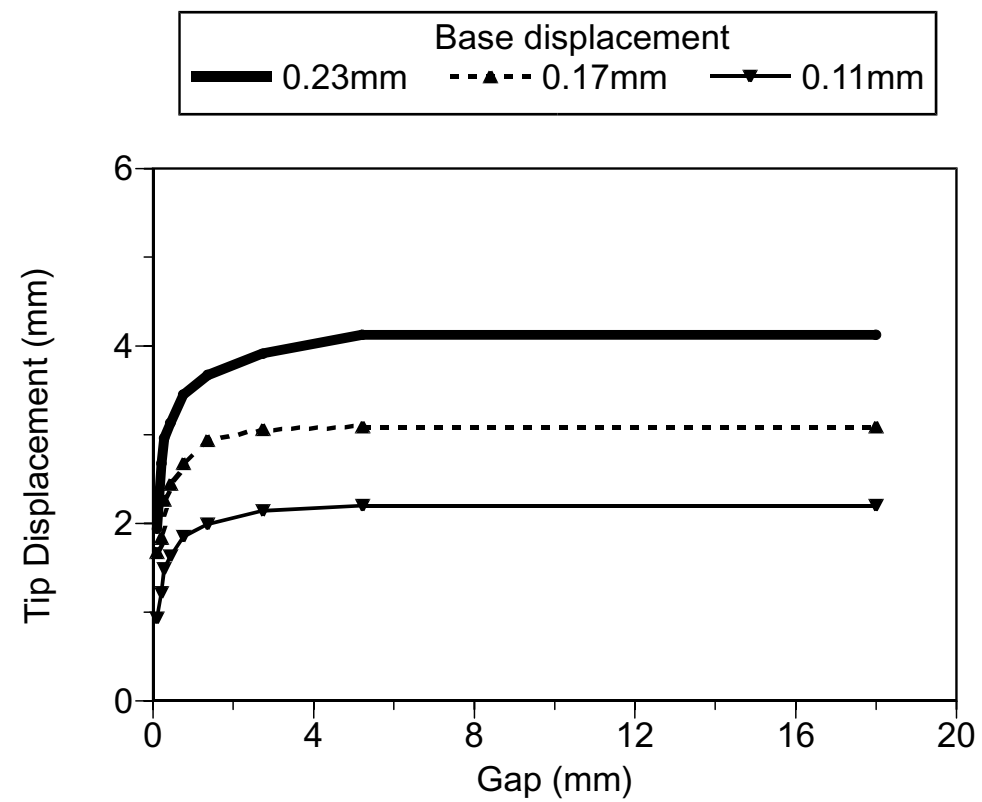

Fig. 10. Performance of beam/MCLD system gaps with base magnets and ferromagnetic constraining layers.

The experimental performance characteristics of the MCLD treatments of beams is presented. The frequency response of beam/MCLD systems is obtained for different arrangements of the magnetic constraining layers and for various spatial spacing between the magnetic layers. It is found that constraining layers that are placed in a state of magnetic attraction produce high damping characteristics as compared to conventional PCLD. In addition, the obtained results indicate that improved structural damping is achieved by reducing the gap between neighboring layers.

The use of partial MCLD treatment is essential to enhance the performance over a broad frequency range by virtue of the nature of the mode shapes of higher order modes of vibration. Furthermore, the weight and cost of the MCLD treatment can be improved by using magnets only in the base to interact with ferromagnetic constraining layers without compromising the damping characteristics of the treatment.

Finally, it is important however to note that hybrid of the MCLD and ACLD can be viable when the characteristics of the MCLD are to be enhanced by the use of the ACLD. The hybrid configuration will be able to compensate, for example, for performance degradation due to changes in the operating temperature or to improve/shape the frequency response characteristics of the composite assembly.

\section{Acknowledgements}

This work is funded by The U.S. Army Research Office (Grant number DAAH-04-96-1-0317). Special thanks are due to Dr. Gary Anderson, the technical monitor, for his invaluable technical inputs.

\section{References}

[1] A. Baz, Magnetic Constrained Layer Damping, U.S.Patent application, 1997.

[2] A. Baz, Magnetic Constrained Layer Damping, Proc. of 11th Conf. on Dynamics \& Control of Large Structures, Blacksburg, VA, May 1997, pp. 333-344,

[3] A. Baz, Active Constrained Layer Damping, US Patent, 1996, pp. $5,485,053$.

[4] A. Baz and J. Ro, Vibration Control of Plates with Active Constrained Layer Damping, J. of Smart Materials and Structures 5 (1996), 272-280.

[5] R. Gentilman, D.F. Fiore, H.T. Pham, K.W. French and L.J. Bowen, Fabrication and Properties of 1-3 PZT Polymer Composites, Ceramic Transactions 43 (1994), pp. 239-247.

[6] N. Hagood and A. von Flottow, Damping of Structural Vibrations with Piezoelectric Materials and Passive Electrical Networks, J. of Sound and Vibration 146 (1991), 243-268.

[7] M. Ruzzene, J. Oh and A. Baz, Finite Element Modeling of Magnetic Constrained Layer Damping, accepted in Journal of Sound and Vibration, 2000.

[8] W. Shields, J. Ro and A. Baz, Control of Sound Radiation from a Plate into an Acoustic Cavity Using Active Piezoelectric Damping Composites, Proc. Of Smart Materials and Structures Conf., San Diego, CA, March 1997.

[9] C.T. Sun and Y.P. Lu, Vibration Damping of Structural Elements, Prentice Hall, Englewood Cliffs, New Jersey, 1995. 

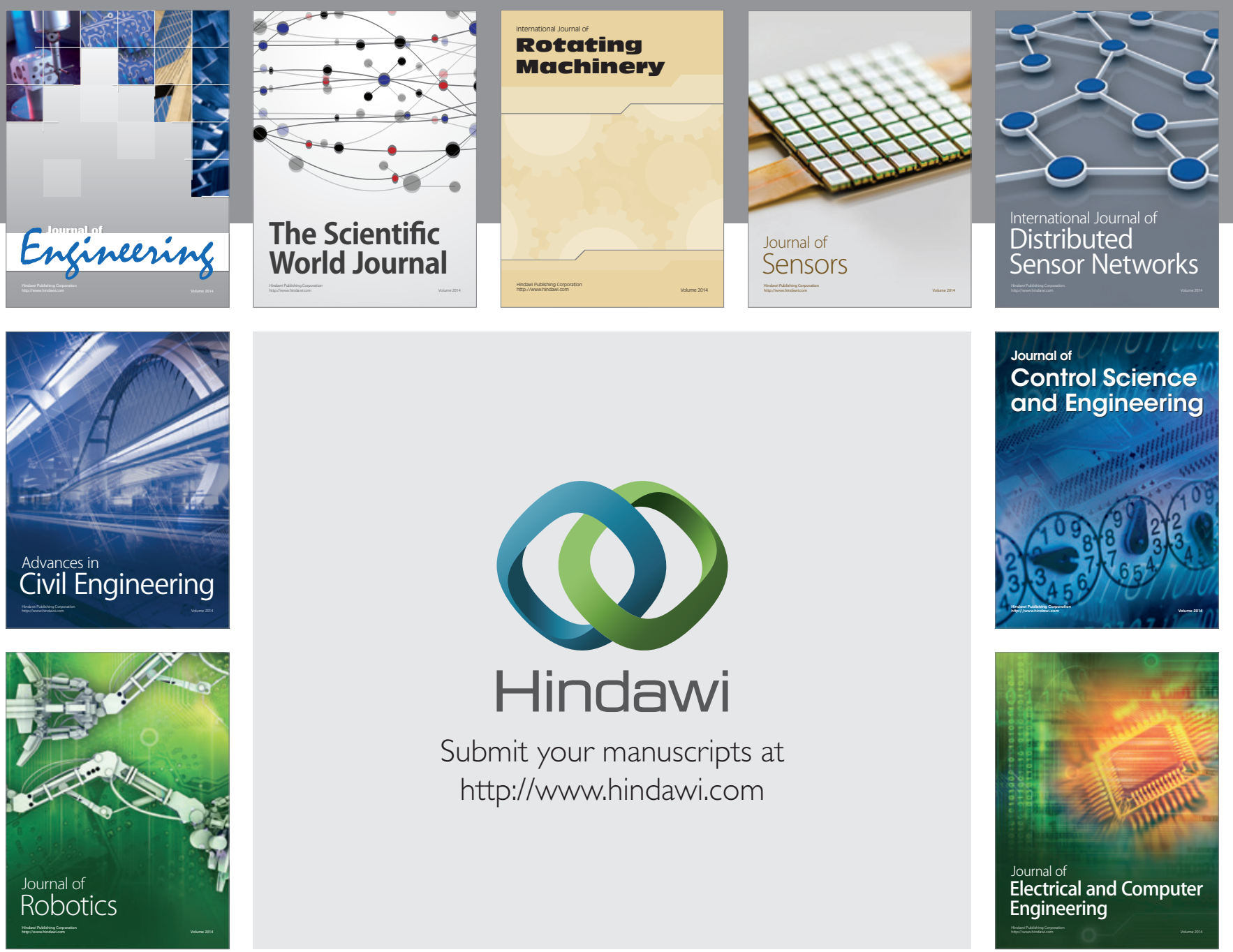

Submit your manuscripts at

http://www.hindawi.com
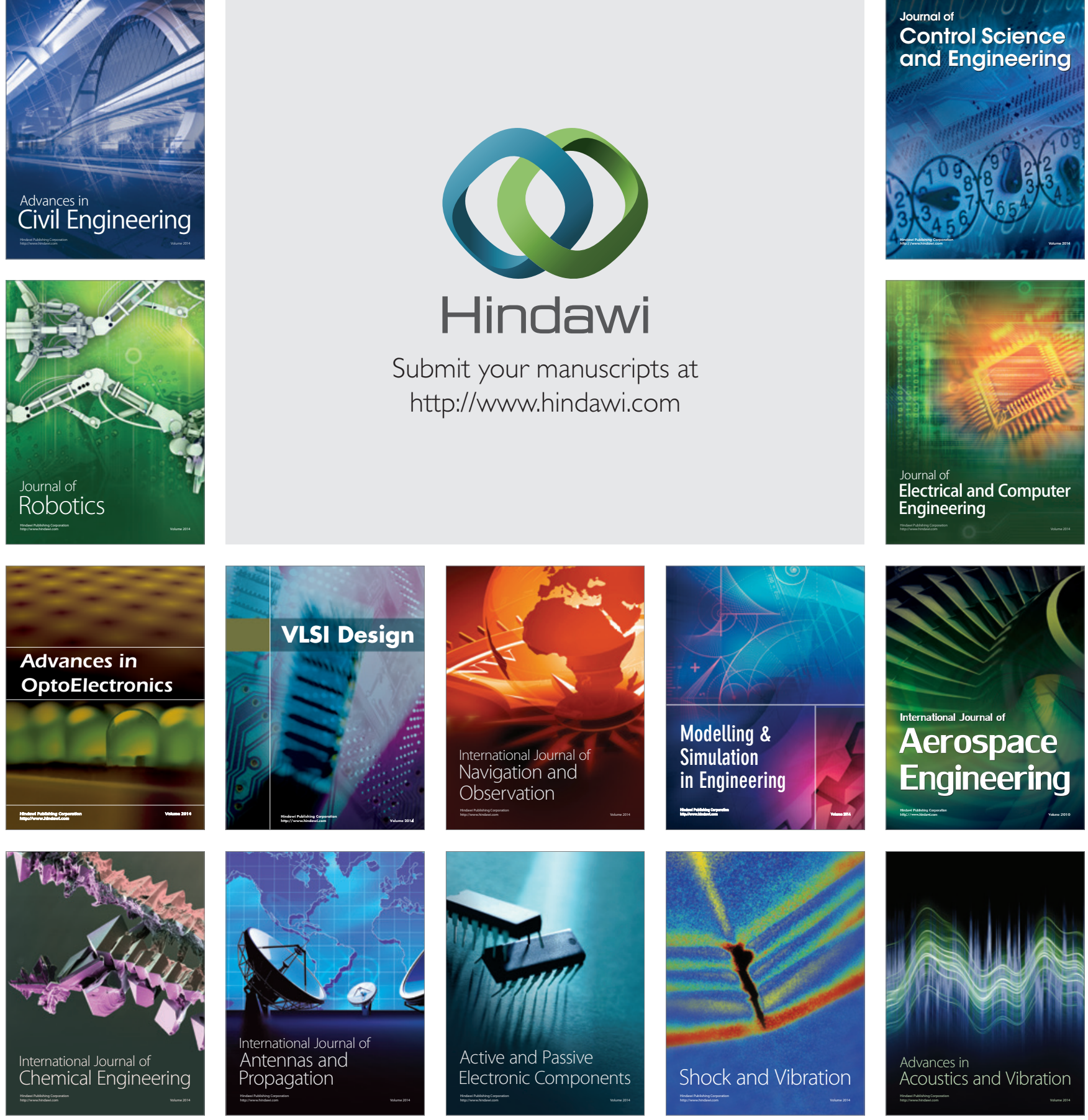